\title{
Genetic Performance of the Semidwarfing Allele sd1 Derived from a Japonica Rice Cultivar and Minimum Requirements to Detect Its Single-Nucleotide Polymorphism by MiSeq Whole-Genome Sequencing
}

\author{
Motonori Tomita (iD ${ }^{1}$ and Kazuo Ishii ${ }^{2}$ \\ ${ }^{1}$ Research Institute of Green Science and Technology, Shizuoka University, Shizuoka 422-8529, Japan \\ ${ }^{2}$ Faculty of Agriculture, Tokyo University of Agriculture and Technology, Fuchu, Tokyo 183-8538, Japan \\ Correspondence should be addressed to Motonori Tomita; tomita.motonori@shizuoka.ac.jp
}

Received 13 April 2017; Revised 3 August 2017; Accepted 16 August 2017; Published 3 April 2018

Academic Editor: Deepmala Sehgal

Copyright (C) 2018 Motonori Tomita and Kazuo Ishii. This is an open access article distributed under the Creative Commons Attribution License, which permits unrestricted use, distribution, and reproduction in any medium, provided the original work is properly cited.

\begin{abstract}
The influence of the semidwarfing gene $s d 1$ derived from the rice cultivar Jukkoku (Jukkoku_sd1) and IR8 (IR8_sd1), which contributed to the Green Revolution, $d 60$ from Hokuriku 100, as well as the combination of $s d 1$ and $d 60$ (Jukkoku_sd1 plus $d 60$ and IR8_sd1 plus d60), was investigated using isogenic lines raised by backcrossing with the cultivar Koshihikari. The isogenic lines carrying Jukkoku_sd1, IR8_sd1, $d 60$, Jukkoku_sd1 plus $d 60$, and IR8_sd1 plus $d 60$ had considerably shorter culm lengths than Koshihikari by $19.2 \%, 22.8 \%, 26.0 \%, 45.1 \%$, and $43.4 \%$, respectively. The $s d 1$ plus $d 60$ lines showed additively reduced culms, indicating that the function of $d 60$ was different from $s d 1$. In contrast to the culm reduction, Jukkoku_sd1 showed productive merit with a panicle length of $2.5 \%$ greater than the origin. MiSeq next-generation sequencer was used to optimize a minimum scale to detect Jukkoku_sdl in practical breeding. Mapping with the reference genome of Nipponbare gained the average depths of Koshihikari Jukkoku_sdl and Koshihikari being 9.17 and 7.29, respectively. Comparing the vcf files of the entire genomes of Koshihikari Jukkoku_sdl and the virtual Koshihikari revealed a G to T SNP at position 38,382,746 in the sdl locus on chromosome 1 of Koshihikari, causing a loss-of-function mutation of GA20-oxidase.
\end{abstract}

\section{Introduction}

Semidwarfism of rice improves phenotype (light-interception properties and harvest index), nitrogen responsiveness, and lodging resistance and is thus an important trait worldwide. The International Rice Research Institute produced a cross between Peta, a tall indica variety (culm length, 150-180 cm), characterized by abundant, long hanging leaves, commonly grown in tropical Asia, and Dee-geo-woo-gen (DGWG), a Taiwanese indigenous semidwarf variety, to improve its lodging resistance and light-interception properties. The resulting semidwarf rice variety IR8 (culm length, $90-100 \mathrm{~cm}$ ), developed in 1966, has dramatically improved rice yields and brought the Green Revolution to tropical Asia [1].

The semidwarf trait has also been introduced into rice cultivars grown in other countries. In Japan, Hoyoku,
Shiranui, and Reihou, which are representative main cultivars grown in the Kyushu region, were developed using the indigenous semidwarf cultivar Jukkoku [2]. A series of cultivars, such as Akihikari and Niigata Wase, were developed in the 1970s in the Tohoku region using the semidwarf cultivar Reimei, which was induced by gammaray irradiation of Fujiminori [3]. In the USA, Calrose 76 (culm length, $\sim 90 \mathrm{~cm}$ ) was developed in 1976 also by gammaray irradiation of the japonica variety Calrose (culm length, $\sim 120 \mathrm{~cm}$ ) [4-6]. In Korea, $d 47$ derived from IR8 was also introduced into Tongil by japonica-indica hybridization [7].

Advances in genetic research have facilitated the identification of genes responsible for semidwarfism in rice. The incomplete recessive gene $d 47$ is responsible for the semidwarfism of DGWG, the parent line of IR8 [8-10]. Later, the incomplete recessive gene $s d 1$ in Calrose 76 was shown 


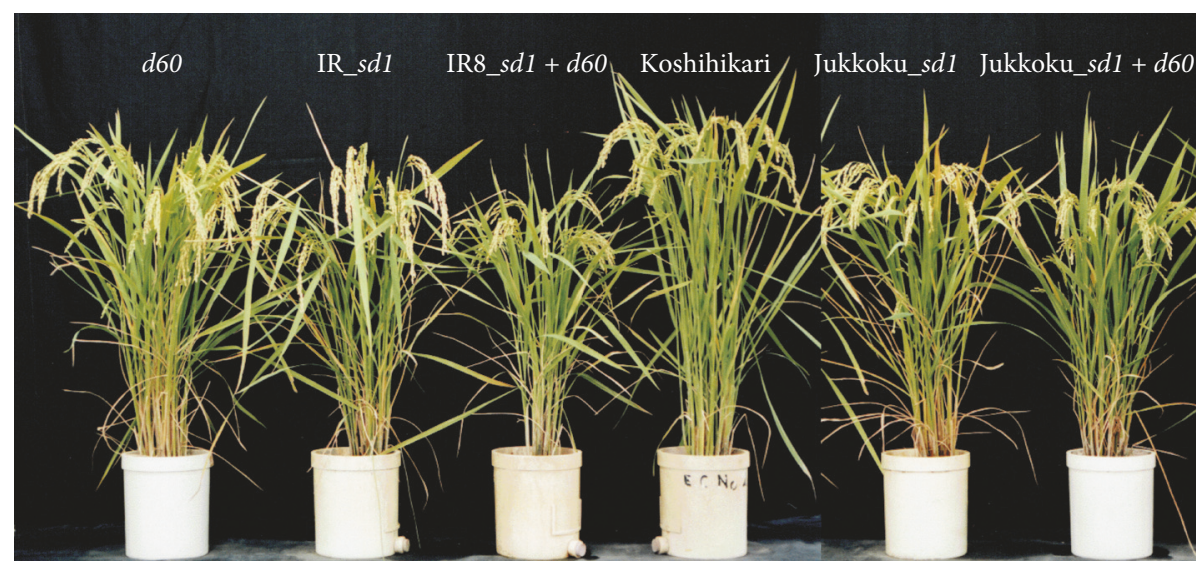

FIGURE 1: Semidwarf isogenic lines with the genetic background of Koshihikari, which contained $d 60$, IR_sd1, IR8_sd1 + d60, Jukkoku_sd1, and Jukkoku_sd1 $+d 60$, respectively.

to be allelic to $d 47[4,11,12]$. Moreover, allelic relationships of the semidwarf gene have been found in Taichung Native 1, derived from DGWG; Shiranui, derived from Jukkoku; and $d 49$ in the mutant cultivar Reimei $[13,14]$. Finally, all these semidwarf cultivars carry alleles at the $s d 1$ locus, despite their different parentage [14-18]. The $s d 1$ alleles, on the long arm of chromosome 1, encode loss-of-function mutations in GA20-oxidase (OsGA20ox2), which regulates the synthesis of biologically active gibberellins (GAs), which catalyze three steps in the GA biosynthesis pathway [15-18]. Taken together, a single semidwarf gene, $s d 1$, solely confers the semidwarf phenotype of cultivars commonly grown around the world due to its nondetrimental effects on grain yield [19].

This narrow genetic base of current semidwarf rice cultivars has led to reduced genetic diversity in rice [20-22]. Thus, it is necessary to identify a novel semidwarf gene alternative to $s d 1$ and to utilize it to extend genetic diversity in semidwarf cultivars. A novel dwarf gene, d60, which was found in the semidwarf mutant Hokuriku 100, developed by exposing Koshihikari to $20 \mathrm{kR}$ of gamma radiation [23,24], is thus of particular importance. Unlike $s d 1$, which is inherited as a single recessive gene, $d 60$ complements the gametic lethal gene gal, which is carried by many rice varieties. Consequently, the cross between Hokuriku 100 and Koshihikari exhibits a unique genotype ratio of 4D60D60: 4D60d60: 1d60d60 [2325].

Although many semidwarf genes allelic to $s d 1$ have been identified in different cultivars (DGWG, Jukkoku, Reimei, and Calrose 76), differences in their influences have not been elucidated. Thus, investigating the differences in ecological and phenotypic traits in relation to yield between 160 - and $s d 1$-carrying plants, as well as those among $s d 1$ allelic variants of different origins, will be beneficial for future use of $d 60$ and multiple allelic variants of $s d 1$. The first author developed isogenic semidwarf lines by continuously backcrossing $s d 1$ of Jukkoku (Jukkoku_sd1) and $s d 1$ of Kinuhikari (IR8_sd1) and $d 60$ and both genes into the Koshihikari background and maintained the lines to investigate the resulting traits. Hence, the study was conducted with the following specific objectives: (a) investigate and quantify the differences between $d 60$ - and $s d 1$-carrying NILs, in relation to yield, to assess the utility of $s d 1$ and $d 60$ semidwarf genes and (b) sequence NILs by MiSeq to detect the differences between $S d 1 / s d 1$ target sites.

\section{Materials and Methods}

2.1. Plant Genetic Materials. As shown in Figure 1, the following rice cultivars were examined: Koshihikari, Koshihikari Jukkoku_sd1 (Koshihikari*6//Koshihikari/Jukkoku $\mathrm{B}_{6} \mathrm{~F}_{4}$ ), Koshihikari IR8_sd1 (Koshihikari*3//Koshihikari/ Kinuhikari $\mathrm{B}_{3} \mathrm{~F}_{3}$, Kinuhikari carrying $s d 1$ of IR8 [26]), Koshihikari $d 60$ (Koshihikari*3//Koshihikari/Hokuriku $100 \mathrm{~B}_{3} \mathrm{~F}_{3}$ ), Koshihikari Jukkoku_sd1 plus $d 60$ (Koshihikari Jukkoku_sd1/Koshihikari d60), and Koshihikari IR8_sd1 plus $d 60$ (Koshihikari IR8_sd1/Koshihikari d60). In each backcross experiment, short-culm $\mathrm{BC}_{n} \mathrm{~F}_{2}$ plants were backcrossed with Koshihikari as the recurrent female parent in each generation. Each semidwarf phenotype was fixed by the $\mathrm{BC}_{n} \mathrm{~F}_{3}$ generation. The percentage of genetic materials from Koshihikari in the isogenic semidwarf lines was calculated as follows: For Koshihikari carrying Jukkoku_sd1 $\left(\mathrm{B}_{6} \mathrm{~F}_{4}\right),\left(1-(1 / 2)^{6}\right) \times 100 \approx 98.4 \%$. For $\mathrm{B}_{3} \mathrm{~F}_{3},\left(1-(1 / 2)^{3}\right) \times 100$ $=87.5 \%$.

2.2. Cultivation. Rice seeds were collected from stocks kept in a refrigerator. Seeds of each line were immersed in just enough water to cover them. Water was changed every day for 7 days (May 2 to May 8) during germination stimulation. Seedlings were grown in special boxes $(30 \times 15 \times$ $3 \mathrm{~cm}$ ) for approximately 20 days in a greenhouse: two seeds were planted in each cell $(2 \times 2 \times 3 \mathrm{~cm})$ in the box on May 11 and 12 and watered twice a day (07:00 and 12:00). Seedlings were then individually transplanted into a paddy field $\left(120 \mathrm{~m}^{2}: 6.0 \times 20.0 \mathrm{~m}\right)$ of the University Farm on June 8. The paddy field received $4.0 \mathrm{~kg}$ of basal fertilizer containing nitrogen, phosphorus, and potassium (weight ratio, nitrogen $:$ phosphorus : potassium $=2.6: 3.2: 2.6)$. Except for the period of mid-season drainage (July 10 to July 17 ), the water level was maintained at $5-7 \mathrm{~cm}$ above ground to prevent seedlings from submersing. A herbicide (Joystar L floable) 
was applied on June 20 to kill weeds growing uncontrollably and the water was then kept at a high enough level to cover the weeds for 1 week. Koshihikari, Koshihikari carrying Jukkoku_sd1, Koshihikari carrying IR8_sdl, Koshihikari carrying $d 60$, Koshihikari carrying $d 60$ and Jukkoku_sdl, and Koshihikari carrying $d 60$ and IR8_sdl were grown in $4 \mathrm{~m}^{2}$ plots $(2 \times 2 \mathrm{~m})$ (two instances per line). Basal fertilizer was applied to give $0.43 \mathrm{~g} / \mathrm{m}^{2}$ nitrogen, $0.53 \mathrm{~g} / \mathrm{m}^{2}$ phosphorus, and $0.53 \mathrm{~g} / \mathrm{m}^{2}$ potassium. After ripening, 10 plants per genotype were sampled twice to assess the following traits: panicle heading time, culm length, internode interval, and panicle length.

2.3. Investigation of Agronomic Traits. The time when the tip of the panicle first emerged from the flag leaf sheath was recorded as the heading time for all plants. Ten plants typical of each line were selected. The sampling procedure was performed twice. Sampled plants were air-dried and the following phenotypic traits were assessed or measured: (1) culm length (the length between the ground surface and the panicle end of the main culm), (2) internode length (the lengths between two neighboring internodes of the upper five internodes), and (3) panicle length (the length between the panicle base and the tip of the panicle). Differences in phenotypic traits between each line carrying a semidwarf gene (or genes) and Koshihikari were obtained using the following equation: percent difference $=[$ (measurements of each line) - (measurements of Koshihikari)]/(measurements of Koshihikari) $\times 100$.

2.4. Next-Generation Sequencing (NGS) Analysis. An advantage of genomics is the development of a next-generation sequencer that can decode DNA sequences at the giga level. Development of the next-generation DNA sequencer was advanced under the Affordable Care Act aims to realize societal implementation of medical genomics $[27,28]$. The desktop-type next-generation sequencer MiSeq has the ability to read 15 million DNA sequences in one run. Generally, whole-genome sequencing analysis required 30 times of the given genome size ( $30 \times$ genome coverage). According to this standard scale, the MiSeq treats only one rice sample per run, because the 15 million DNA amount is just enough to reconstruct a single rice genome. However, it is a big problem that the running cost is excessively high to use the MiSeq at the practical breeding to detect target genes. So, in this study, to achieve a minimum scale to detect target genes with a reasonable cost, we try to detect Jukkoku_sd1 at only $5 \times$ rice genome coverage by using the MiSeq. The semidwarf gene $s d 1$ (a loss-of-function mutation of the GA20-oxidase encoding gene) was transferred to Koshihikari by consecutive backcrosses to prepare a semidwarf Koshihikari named Koshihikari Jukkoku_sdl. The Koshihikari Jukkoku_sdl backcross was used to detect single-nucleotide polymorphisms (SNPs) in Jukkoku-derived $s d l$ by NGS. Whole-genome analysis was conducted using Koshihikari Jukkoku_sdl and Koshihikari. Genomic DNA was extracted from each cultivar by the CTAB (hexadecyltrimethylammonium bromide) method. Genomic DNA was tagged and fragmented to an average length of $500 \mathrm{bp}$ using the Nextera ${ }^{\circledR}$

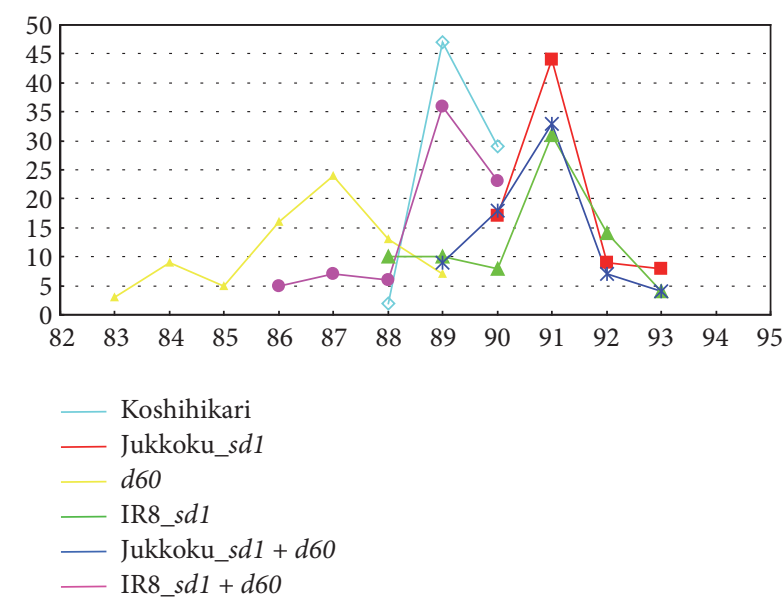

FIgURE 2: Panicle heading time of isogenic lines introgressed with Jukkoku_sd1, IR8_sd1, d60, and their combinations.

transposome-based approach. After purification of the transposome with DNA Clean \& Concentrator ${ }^{\mathrm{TM}}-5$ (Zymo Research, Irvine, CA, USA), adaptors for fixation on the flow cell were synthesized at both ends of each fragment using a polymerase chain reaction (PCR). Then, the DNA fragments were subjected to size selection using AMPure XP magnetic beads (Beckman Coulter, Inc., Brea, CA, USA). Finally, qualitative and quantitative measurements were performed using a Fragment Analyzer ${ }^{\mathrm{TM}}$ (Advanced Analytical Technologies, Inc., Ankeny, IA, USA) and a Qubit ${ }^{\circledR} 2.0$ Fluorometer (Thermo Fisher Scientific, Waltham, MA, USA) to prepare a DNA library for NGS. Aiming to achieve $5 \times$ rice genome coverage, a MiSeq next-generation sequencer was used to simultaneously analyze five lines; namely, $4-5 \mathrm{ng}$ of five DNA libraries was applied in each MiSeq run. Clusters then were formed on the flow cells by bridge-PCR and each pair-end of a 250 bp read was sequenced. Resulting sequenced reads were mapped using BWA software with the Nipponbare genome [29] as a reference. Then, SNPs and Indels were detected using SAMtools software (http://samtools.sourceforge.net/).

\section{Results}

3.1. Effects of Semidwarf Genes sd1 and $d 60$ on Heading Time. The days to heading were compared (Figure 2). The earliness of varieties of Koshihikari and donors of semidwarf genes is as follows: Koshihikari, an early-medium maturing variety; Jukkoku, a medium-late maturing variety; Kinuhikari, an early-medium maturing variety; and Hokuriku 100, an early-medium maturing variety. The heading panicles were observed first in Koshihikari carrying IR8_sdl (83 days to heading) and the latest heading panicles were observed in Koshihikari carrying Jukkoku_sdl or Jukkoku_sdl plus $d 60$ (93 days to heading). However, the difference in the average number of days to heading was the largest between lines carrying IR8_sd1 (86.5 days) and those carrying $d 60$ (90.5 days), but this 4-day difference was thought to be minor (Figure 2). Thus, it was concluded that the time required for maturing was comparable among lines and the differences 
TABLE 1: Effects of the semidwarfing genes Jukkoku_sd1, IR8_sd1, and $d 60$ and their combinations on panicle length, culm length, and internode interval.

\begin{tabular}{|c|c|c|c|c|c|c|c|}
\hline Gene & $\begin{array}{c}\text { Panicle } \\
\text { length }(\mathrm{cm})\end{array}$ & $\begin{array}{l}\text { Culm length } \\
(\mathrm{cm})\end{array}$ & $\begin{array}{l}\text { 1st internode } \\
\text { interval }(\mathrm{cm})\end{array}$ & $\begin{array}{c}\text { 2nd } \\
\text { internode } \\
\text { interval }(\mathrm{cm})\end{array}$ & $\begin{array}{l}\text { 3rd internode } \\
\text { interval }(\mathrm{cm})\end{array}$ & $\begin{array}{l}4 \text { th internode } \\
\text { interval }(\mathrm{cm})\end{array}$ & $\begin{array}{l}\text { 5th internode } \\
\text { interval }(\mathrm{cm})\end{array}$ \\
\hline Koshihikari & 16.2 & 88.8 & 39.7 & 20.7 & 17.8 & 11.6 & 3.1 \\
\hline Jukkoku_sdl & $\begin{array}{c}16.6 \\
(+2.5 \%)\end{array}$ & $\begin{array}{c}71.8 \\
(-19.2 \%)\end{array}$ & $\begin{array}{c}30.2 \\
(-23.8 \%)\end{array}$ & $\begin{array}{c}15.0 \\
(-27.5 \%)\end{array}$ & $\begin{array}{c}13.0 \\
(-27.0 \%)\end{array}$ & $\begin{array}{c}8.8 \\
(-23.3 \%)\end{array}$ & $\begin{array}{c}2.5 \\
(-19.4 \%)\end{array}$ \\
\hline IR8_sd1 & $\begin{array}{c}15.8 \\
(-2.4 \%)\end{array}$ & $\begin{array}{c}68.5 \\
(-22.8 \%)\end{array}$ & $\begin{array}{c}33.2 \\
(-16.4 \%)\end{array}$ & $\begin{array}{c}14.2 \\
(-31.5 \%)\end{array}$ & $\begin{array}{c}11.2 \\
(-37.0 \%)\end{array}$ & $\begin{array}{c}6.6 \\
(-42.6 \%)\end{array}$ & $\begin{array}{c}1.7 \\
(-45.0 \%)\end{array}$ \\
\hline$d 60$ & $\begin{array}{c}15.7 \\
(-3.0 \%)\end{array}$ & $\begin{array}{c}65.7 \\
(-26.1 \%)\end{array}$ & $\begin{array}{c}30.1 \\
(-24.2 \%)\end{array}$ & $\begin{array}{c}14.0 \\
(-32.6 \%)\end{array}$ & $\begin{array}{c}12.9 \\
(-27.4 \%)\end{array}$ & $\begin{array}{c}7.6 \\
(-34.5 \%)\end{array}$ & $\begin{array}{c}1.7 \\
(-45.7 \%)\end{array}$ \\
\hline Jukkoku_sd $1+d 60$ & $\begin{array}{c}15.8 \\
(-2.2 \%)\end{array}$ & $\begin{array}{c}48.6 \\
(-45.3 \%)\end{array}$ & $\begin{array}{c}26.7 \\
(-32.7 \%)\end{array}$ & $\begin{array}{c}10.7 \\
(-48.5 \%)\end{array}$ & $\begin{array}{c}7.9 \\
(-55.8 \%)\end{array}$ & $\begin{array}{c}3.1 \\
(-72.8 \%)\end{array}$ & $\begin{array}{c}0.5 \\
(-83.5 \%)\end{array}$ \\
\hline IR8_sd1 $+d 60$ & $\begin{array}{c}15.7 \\
(-3.3 \%) \\
\end{array}$ & $\begin{array}{c}50.2 \\
(-43.5 \%) \\
\end{array}$ & $\begin{array}{c}30.1 \\
(-24.3 \%) \\
\end{array}$ & $\begin{array}{c}11.2 \\
(-46.0 \%)\end{array}$ & $\begin{array}{c}6.6 \\
(-63.0 \%)\end{array}$ & $\begin{array}{c}1.9 \\
(-83.6 \%)\end{array}$ & $\begin{array}{c}0.3 \\
(-91.8 \%)\end{array}$ \\
\hline
\end{tabular}

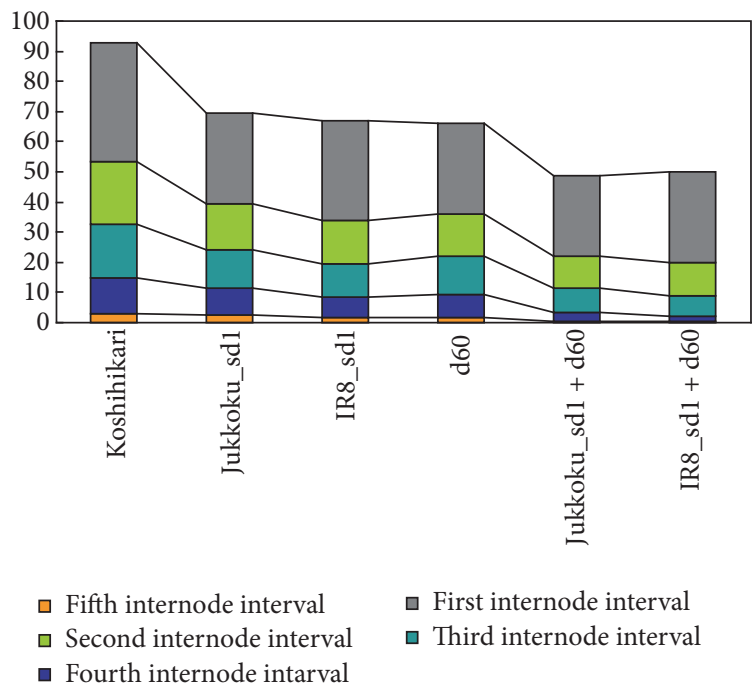

FIGURE 3: Effects of the semidwarfing genes Jukkoku_sd1, IR8_sd1, and $d 60$ and their combinations on internode constitution.

in traits, such as panicle length, were attributed to genetic factors.

\subsection{Effects of Semidwarf Genes sd1 and $d 60$ on Traits Related to} Plant Type. The mean values of the individual traits in plants of each genotype were calculated and the percent differences relative to the corresponding mean values of Koshihikari were compared. Introduction of a semidwarf gene (or genes) resulted in a reduction in culm length. The mean culm length of Koshihikari was $88.8 \mathrm{~cm}$, while those of lines carrying Jukkoku_sd1, IR8_sd1, $d 60$, Jukkoku_sd1 plus $d 60$, and IR8_sd1 plus $d 60$ were $71.8,68.5,65.7,48.6$, and $50.2 \mathrm{~cm}$, respectively (Table 1 and Figure 3). Similarly, internode intervals were also reduced by the introduction of at least one semidwarf gene. While reductions in internode intervals were relatively uniform in lines carrying Jukkoku_sd1 (23.9\%, 27.5\%, 27.0\%, $23.3 \%$, and $19.4 \%$ : percent difference, from upper to lower internode intervals), the reduction in length between the

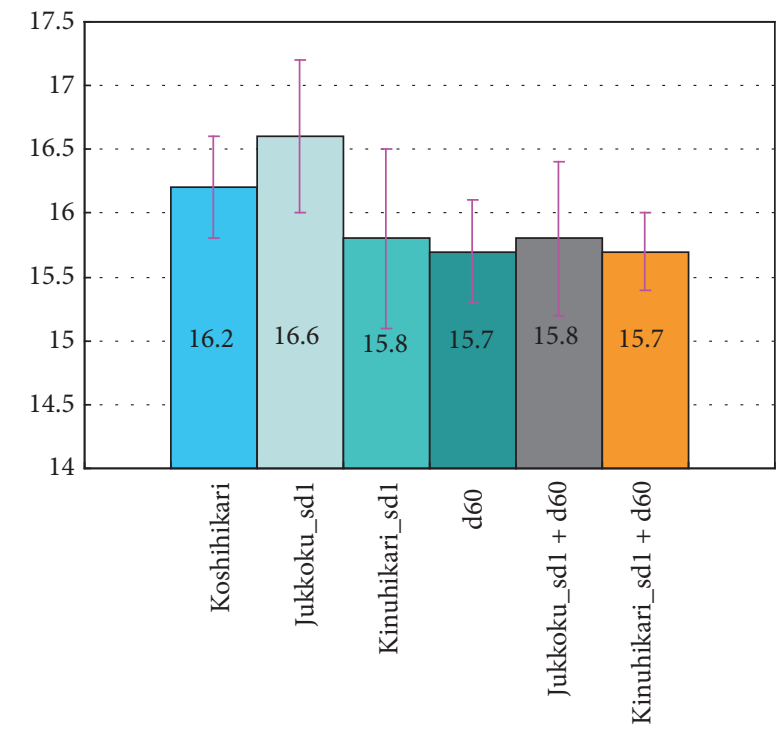

FIGURE 4: Effects of the semidwarfing genes Jukkoku_sdl, IR8_sdl, and $d 60$ and their combinations on panicle length.

third and fourth and between the fourth and fifth internodes was markedly large in lines carrying IR8_sd1 (16.4\%, 31.5\%, $37.0 \%, 42.6 \%$, and $45.0 \%$ : percent difference, from upper to lower internode intervals). Reductions in internode intervals were relatively uniform in lines carrying $d 60$ (24.2\%, 32.6\%, $27.4 \%, 34.5 \%$, and $45.7 \%$ : percent difference, from upper to lower internode intervals), while percent differences were larger than those observed in lines carrying Jukkoku_sdl. In the $s d 1$ plus $d 60$ lines, marked reductions were observed in length between neighboring internodes, probably attributed to the additive effect of $s d 1$ and $d 60$ (Table 1 and Figure 3). The interval between the fourth and fifth internodes often disappeared in plants carrying IR8-sd1 and in $s d 1$ plus $d 60$ lines, which showed a marked reduction in length between the lower internodes.

In contrast, as shown in Figure 4, the effect of a semidwarf gene (or genes) on panicle length $(-3.0$ to $+2.5 \%)$, which 


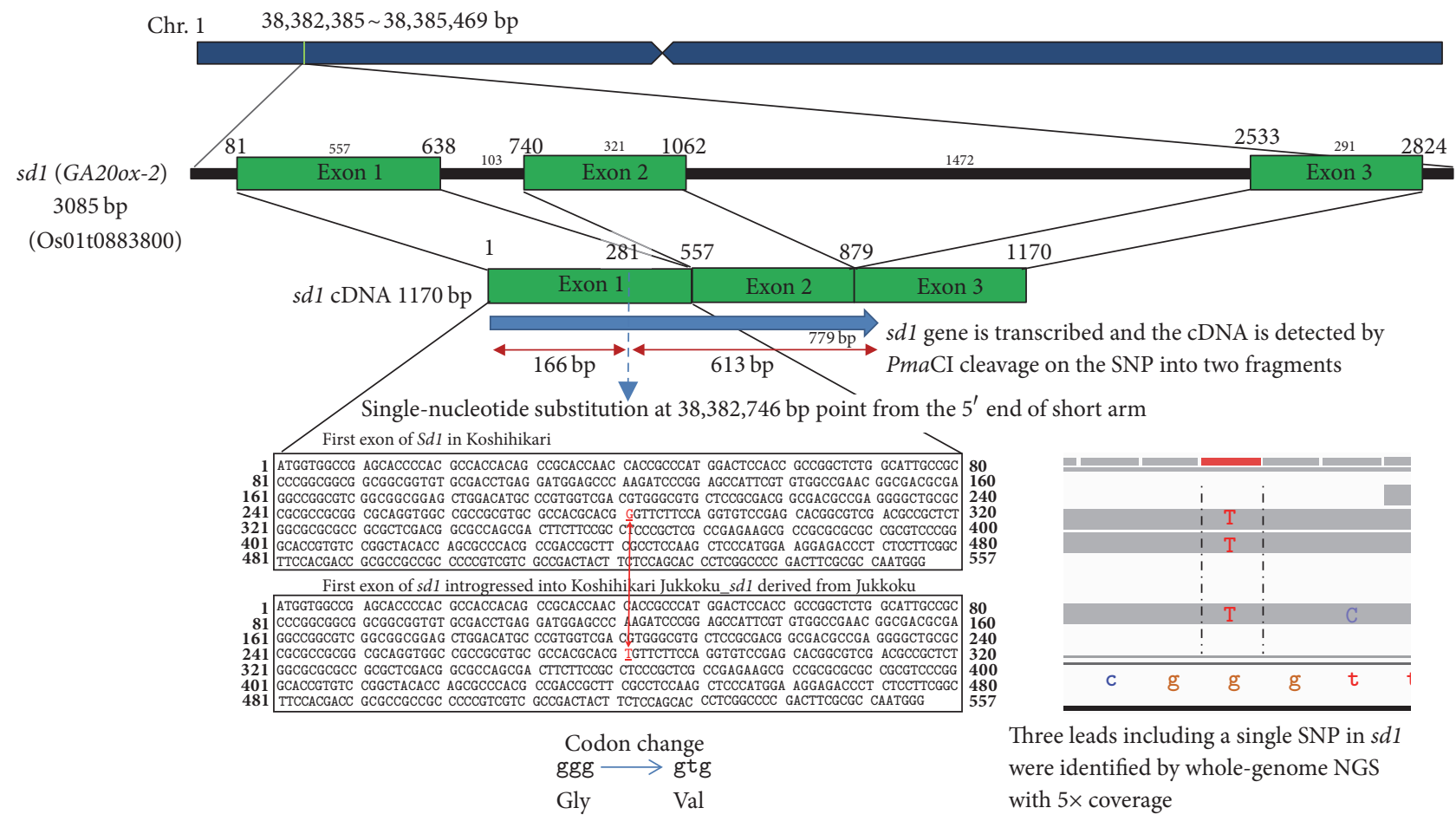

FIGURE 5: SNP in Jukkoku-derived $s d 1$ identified by whole genomic NGS for the genome of Koshihikari Jukkoku_sd1 with coverage 5×.

was smaller than that for internode intervals ( -16 to $-92 \%)$, was negligible in practical agriculture. Particularly, the mean length of panicles in the line carrying Jukkoku_sdl was $16.2 \mathrm{~cm}$, which was solely $2.5 \%$ longer than that of Koshihikari. The panicle length of the line carrying Jukkoku_sdl was longer than that of the original cultivar Koshihikari: not only the mean value but also the value including the standard deviation caused by circumstance. Therefore, there was certain merit in increasing the production of Jukkoku_sd1 as compared to other semidwarf genes.

3.3. NGS Analysis to Detect Jukkoku-Derived sdl with a Minimum Scale. Using the MiSeq sequencer, we obtained a total read length of $2.79 \times 10^{9} \mathrm{bp}$ from the total read number of $9.53 \times 10^{6}$ in Koshihikari Jukkoku_sdl, while a total read length of $1.92 \times 10^{9} \mathrm{bp}$ was obtained from a total read number of $6.13 \times 10^{6}$ in Koshihikari. By mapping the read sequences obtained by NGS using Nihonbare genomic DNA as a reference, sequence coverage rates of $93.5 \%$ and $88.5 \%$ were attained for Koshihikari Jukkoku_sd 1 and Koshihikari, respectively, while average depths were 9.17 and 7.29 , respectively. Furthermore, vcf files of the entire genomes were prepared and the whole-genome sequence of Koshihikari Jukkoku_sdl was compared with that of the virtual Koshihikari genome. As a result, three reads were obtained, including Jukkoku_sdl from Koshihikari Jukkoku_sd1, while three reads of $S d 1$ were obtained from Koshihikari. The $S d 1 / s d 1$ locus (Os01t0883800-01) was localized at positions $38,382,385-38,385,469$ from the end of the short arm of chromosome 1 in the Koshihikari genome. The difference observed between the Koshihikari_Sd1/Jukkoku_sd1 alleles was only one SNP from $G$ to $T$ in exon 1 of the GA20oxidase gene (Figure 5), as reported by Sasaki et al. [17]. In this study, the G to T SNP of the defective GA20-oxidase gene was localized at position 38,382,746 from the end of the short arm of chromosome 1 of Koshihikari. Using this scale of the NGS approach with a coverage of only $5 x$, the targeted SNP in Jukkoku_sd1 was successfully identified with three reads, with a cost reduced to $1 / 5$ that of the ordinal whole-genome sequencing with a coverage of $30 \times$.

\section{Discussion}

Environmental degradation caused by global warming, postearthquake salt damage, and radioactive contamination and globalization of agricultural markets due to the TransPacific Partnership are serious issues that call for the innovation of new cultivars to overcome the shortcomings of current crops. The results of this study showed that all tested semidwarf lines had shorter culm lengths than Koshihikari, indicating improved lodging resistance. Furthermore, the leaves were straighter (pointing upwards) in the semidwarf lines than in Koshihikari (Figure 1), indicating improved light-interception properties attributed to the introduction of semidwarf gene(s). Panicle length was solely longer by $2.5 \%$ in Koshihikari carrying Jukkoku_sdl and shorter in lines carrying IR8_sd $d$ by $2.4 \%$ or $d 60$ by $3.0 \%$, as compared with the original cultivar Koshihikari (Table 1 and Figure 4). However, the reduction in panicle length was considerably less than that in culm length (Table 1 and Figure 3; a 22.8\% decrease in lines carrying IR8_sdl versus a $26.1 \%$ decrease in lines carrying $d 60$ ), suggesting that the negative effects 
of the semidwarf genes $s d 1$ and $d 60$ on panicle length were negligible (Table 1 and Figure 4). Ogi et al. [30] and Murai et al. [31] reported that an isogenic line carrying $s d 1$ was derived from DGWG, which was constructed with the genetic background of Norin 29 and Shiokari, respectively. However, IR8-derived $s d 1$ did not show effects of increasing panicle length, which was also observed with the genetic background of Koshihikari in this study. On the other hand, the results showed that Jukkoku-derived $s d 1$ solely increased panicle length by more than $2.5 \%$, as compared to the original cultivar Koshihikari and other semidwarfing alleles.

The first author reported transcription of the $s d l$ gene derived from Jukkoku [32]. RT-PCR of root RNA showed that the $779 \mathrm{bp}$ fragment derived from the $s d 1$ locus was clearly cleaved into $613 \mathrm{bp}$ and $166 \mathrm{bp}$ fragments by PmaCI digestion on the SNP in Jukkoku_sdl, but there was no cleavage of the $S d 1$ locus in Koshihikari (Figure 5). This is the first evidence of the transcription of $s d l$, a defective gene of GA20ox2. Accordingly, Jukkoku_sdl, which is substituted by only a single nucleotide against the $385 \mathrm{bp}$-deficient IR_sd1 [17] and is actively transcribed, has no deteriorative effect on the panicle and may have a positive effect on panicle elongation by the overflow of nutrition due to culm reduction.

In Japan, Koshihikari suffers considerable lodging damage as a result of frequent powerful typhoons and, thus, the development of lodging-resistant cultivars has been a longstanding challenge. The first author transferred the semidwarf gene Jukkoku_sd1 to Koshihikari to develop a semidwarf form of Koshihikari which could withstand a typhoon by backcrossing with Koshihikari eight times [33], with more than $99.8 \%$ of the background of the Koshihikari genome, except for Jukkoku_sd1 [32]. This cultivar, which was about $20 \mathrm{~cm}$ shorter than Koshihikari, was named Hikarishinseiki (rice cultivar number 12273) [33,34]. Hikarishinseiki is the first cultivar to be a Koshihikari-type semidwarf with $s d 1$ registered in Japan and USA $[35,36]$.

In this study, a MiSeq next-generation sequencer was used to achieve $5 \times$ rice genome coverage; namely, $4-5 \mathrm{ng}$ of five DNA libraries was applied in one MiSeq run. Using this approach, the targeted gene mutation in Jukkoku_sdl was successfully detected as the SNP (G to T) in the defective GA20-oxidase gene, which had reduced the culm length by a loss-of-function mutation of GA synthesis, localized at position 38,382,746 from the end of the short arm of chromosome 1 of Koshihikari. This would be the minimum scale to detect Jukkoku_sdl in practical breeding. In Japan, genetically modified organisms are not acceptable to consumers; thus the target SNP in Jukkoku_sd1 would be effectively tracked by using NGS of $5 \times$ coverage scale in each backcrossed generation with Koshihikari. On the other hand, as the DNA sequence and function of $s d 1$ have been deciphered [1518, 37], new breeding methods, such as RNAi gene silencing [38] and genome editing [39], are available in other countries to retard culm length by knockout of $S d 1$.

The effects on phenotypic traits of rice differed between the two semidwarf genes ( $s d 1$ and $d 60$ ) and also between $s d 1$ loci of different origins (Jukkoku_sd1 and IR8_sd1). The effect on culm length was more pronounced in plants carrying $d 60$ than in those carrying $s d 1$ (culm length: Jukkoku_sdl,
$71.8 \mathrm{~cm}$; IR8_sd1, $68.5 \mathrm{~cm}$; and $d 60,65.7 \mathrm{~cm}$ ). The reduction in internode intervals was relatively uniform in lines carrying Jukkoku_sdl or $d 60$ (uniform reduction type), while reductions of the third and lower intervals were larger than in the upper intervals in lines carrying IR8_sdl (Figure 3 and Table 1). Thus, the center of gravity will be lower in the lower internode reduction type, suggesting higher lodging resistance in lines carrying IR8_sdl than in those carrying Jukkoku_sd1 or $d 60$. Although the function of $d 60$ is not known, it is clearly distinct from that of $s d l$, as the additive double-dwarf effect of $s d 1$ and $d 60$ appeared in Jukkoku_sd1 plus $d 60$ and IR8_sd1 plus $d 60$, respectively. The results of this study demonstrated that $d 60$ has a stronger effect than $s d 1$ on culm length and exerts similar effects on other phenotypic traits as with $s d 1$. Although many semidwarf genes are associated with a reduction in panicle length, $d 60$ does not exert such negative effects on phenotypic traits of rice plants. Taken together, these results showed that $d 60$ is useful for adding genetic diversity to semidwarf varieties and is thus of particular importance in the field of plant bleeding.

\section{Conflicts of Interest}

The authors have no conflicts of interest to declare.

\section{Acknowledgments}

This work was funded by a grant from the Adaptable and Seamless Technology Transfer Program (A-STEP) through Target-Driven R\&D (high-risk challenge type) by Japan Science and Technology Agency to Motonori Tomita, whose project is titled "NGS Genome-Wide Analysis-Based Development of Rice Cultivars with Super High-Yield, LargeGrains, and Early/Late Flowering Suitable for the Globalized World and Global Warming," from 2014 to 2018.

\section{Supplementary Materials}

DNA sequences of $S d 1 / s d 1$ locus. (Supplementary Materials)

\section{References}

[1] G. S. Khush, "Green revolution: preparing for the 21st century," Genome, vol. 42, no. 4, pp. 646-655, 1999.

[2] M. Okada, Y. Yamakawa, K. Fujii et al., "On the new varieties of paddy rice, "Hoyoku", "Kokumasari" and "Shiranui"," Hoyoku, Kokumasari and Shiranui , Bulletin of Kyushu Agricultural Experimental Station, vol. 12, no. 3, pp. 187-224, 1967.

[3] Y. Futuhara, "Breeding of new rice variety Reimei by gamma ray irradiation," Gamma Field Symposia, vol. 7, pp. 87-109, 1968.

[4] J. N. Rutger, M. L. Peterson, C. H. Hu, and W. F. Lehman, "Induction of useful short stature and early maturing mutants in two japonica rice cultivars," Crop Science, vol. 16, no. 5, pp. 631-635, 1976.

[5] J. N. Rutger, M. L. Peterson, and C.-H. Hu, "Registration of Calrose76 rice," Crop Science, vol. 17, p. 978, 1977.

[6] J. N. Rutger, "Applications of induced and spontaneous mutation in rice breeding and genetics," Advances in Agronomy, vol. 36, no. C, pp. 383-413, 1983. 
[7] G.-S. Chung and M.-H. Heu, "Status of japonica-indica hybridization in Korea," in Innovative Approaches to Rice Breeding, pp. 135-152, IRRI, Los Banos, Philippines, 1980.

[8] T. T. Chang, H. Morishima, C.-S. Huang, T. Tagumpay, and K. Takeo, "Genetic analysis of plant height, maturing and other quantitative traits in the cross of Peta $\times$ I-geo-tze," Journal of Agricultural Association in China, vol. 39, pp. 1-8, 1965.

[9] R. C. Aquino and P. R. Jennings, "Inheritance and Significance of Dwarfism in an Indica Rice Variety," Crop Science, vol. 6, pp. 551-554, 1966.

[10] H. H. Mun, T. Chang, and H. Beachell, "The inheritance of culm length, panicle length, duration to heading and bacterial leaf blight reaction in rice cross: Sigadis $\times$ Taichung (Native)," Japanese Journal of Breeding, vol. 18, no. 1, pp. 7-11, 1968.

[11] K. W. Foster and J. N. Rutger, "Inheritance of semidwarfism in rice, Oryza sativa L," Genetics, vol. 88, no. 1, pp. 559-574, 1978.

[12] D. J. Mackill and J. N. Rutger, "The inheritance of inducedmutant semidwarfing genes in rice," Journal of Heredity, vol. 70, no. 5, pp. 335-341, 1979.

[13] H. Ikehashi and F. Kikuchi, "Genetic analysis of semidwarfness and their significance for breeding of high-yielding varieties in rice," J.A.R.Q, vol. 15, pp. 231-235, 1982.

[14] F. Kikuchi and Y. Futsuhara, "Inheritance of morphological characters. 2. Inheritance of semidwarf in," in Science of the Rice Plant, T. Matsuo, S. Shimizu, S. Tsunoda et al., Eds., vol. 3, pp. 309-317, Tokyo Food and Agricultural Policy Research Center, Tokyo, Japan, 1997.

[15] L. Monna, N. Kitazawa, R. Yoshino et al., "Positional cloning of rice semidwarfing gene, $s d-1$ : Rice "green revolution gene" encodes a mutant enzyme involved in gibberellin synthesis," DNA Research, vol. 9, no. 1, pp. 11-17, 2002.

[16] W. Spielmeyer, M. H. Ellis, and P. M. Chandler, "Semidwarf (sd1), "green revolution" rice, contains a defective gibberellin 20oxidase gene," Proceedings of the National Academy of Sciences of the United States of America, vol. 99, no. 13, pp. 9043-9048, 2002.

[17] A. Sasaki, M. Ashikari, M. Ueguchi-Tanaka et al., "A mutant gibberellin-synthesis gene in rice: New insight into the rice variant that helped to avert famine over thirty years ago," Nature, vol. 416, no. 6882, pp. 701-702, 2002.

[18] M. Ashikari, A. Sasaki, M. Ueguchi-Tanaka et al., "Loss-offunction of a rice gibberellin biosynthetic gene, GA20 oxidase (GA20ox-2), led to the rice 'green revolution"' Breeding Science, vol. 52, no. 2, pp. 143-150, 2002.

[19] P. Hedden, "Constructing dwarf rice," Nature Biotechnology, vol. 21, no. 8, pp. 873-874, 2003.

[20] T.-T. Chang and B. S. Vergara, "Ecological and genetic information on adaptability and yielding ability in tropical rice varieties," in Rice Breeding, IRRI, Los Banos, Philippienes, 1972.

[21] T.-T. Chang, C. Zuno, A. Marciano-Romena, and G. C. Loresto, "Semidwarfs in rice improvement," Phytobreedon, vol. 1, p. 1, 1985.

[22] P. Hedden, “The genes of the green revolution," Trends in Genetics, vol. 19, no. 1, pp. 5-9, 2003.

[23] M. Tomita, T. Tanisaka, Y. Okumoto, and H. Yamagata, "Linkage analysis for the gametic lethal gene of rice variety Koshihikari and the semidwarf gene induced in Koshihikari," in Proceedings of the 6th International Congress of the Society for the Advancement of Breeding Researches in Asia and Oceania, S. Iyama and G. Takeda, Eds., The Key to the Survival of the Earth, pp. 345348, Tsukuba, August 1989.
[24] M. Tomita, "The gametic lethal gene gal: activated only in the presence of the semidwarfing gene $\mathrm{d} 60$ in rice," in Rice Genetics III, G. S. Khush, Ed., pp. 396-403, International Rice Research Institute, Manila, Philippines, 1996.

[25] M. Tomita, "Combining two semidwarfing genes d60 and sd1 for reduced height in "Minihikari", a new rice germplasm in the "Koshihikari" genetic background," Genetics Research, vol. 94, no. 5, pp. 235-244, 2012.

[26] H. Tabuchi, N. Hashimoto, A. Takeuchi, T. Terao, and Y. Fukuta, "Genetic analysis of semidwarfism of the japonica rice cultivar Kinuhikari," Breeding Science, vol. 50, no. 1, pp. 1-7, 2000.

[27] E. M. Bahassi and P. J. Stambrook, "Next-generation sequencing technologies: Breaking the sound barrier of human genetics," Mutagenesis, vol. 29, no. 5, pp. 303-310, 2014.

[28] S. E. Levy and R. M. Myers, "Advancements in Next-Generation Sequencing," Annual Review of Genomics and Human Genetics, vol. 17, pp. 95-115, 2016.

[29] T. Sasaki, “The map-based sequence of the rice genome," Nature, vol. 436, no. 7052, pp. 793-800, 2005.

[30] Y. Ogi, H. Kato, K. Maruyama, and F. Kikuchi, “The effects on the culm length and other agronomic characters caused by semidwarfing genes at the sd-1 locus in rice," Japanese Journal of Breeding, vol. 43, no. 2, pp. 267-275, 1993.

[31] M. Murai, T. Komazaki, and S. Sato, "Effects of sd1 and Ur1 (Undulate rachis -1) on lodging resistance and related traits in rice," Breeding Science, vol. 54, no. 4, pp. 333-340, 2004.

[32] M. Tomita and S. Matsumoto, "Transcription of rice green revolution gene $s d 1$ is clarified by comparative RNA diagnosis using the isogenic background," Genomics and Applied Biology, vol. 2, no. 5, pp. 29-35, 2011.

[33] M. Tomita, "Introgression of Green Revolution sd1 gene into isogenic genome of rice super cultivar Koshihikari to create novel semidwarf cultivar 'Hikarishinseiki' (Koshihikari-sd1)," Field Crops Research, vol. 114, no. 2, pp. 173-181, 2009.

[34] MAFF, “Hikarishinseiki varietal registration," Official Gazette 8 Nov. Ministry of Agriculture, Forestry and Fisheries, vol. 8, 2004, Tokyo, Japan.

[35] MAFF, "Hikarishinseiki brand rice description in Okayama and Tottori prefectures," Official Gazette 28 Mar. Ministry of Agriculture, Forestry and Fisheries, 2007, Tokyo, Japan.

[36] M. Tomita, "Hikarishinseiki," Oryza sativa L. Plant Variety Protection Number: 201000072, USDA-AMS-ST-PVPO, Washington, DC, 2013. http://www.ars-grin.gov/cgi-bin/npgs/pvp/ showpvp.pl?pvpno=201000072.

[37] T. Oikawa, M. Koshioka, K. Kojima, H. Yoshida, and M. Kawata, "A role of OsGA20ox1, encoding an isoform of gibberellin 20oxidase, for regulation of plant stature in rice," Plant Molecular Biology, vol. 55, no. 5, pp. 687-700, 2004.

[38] F. Qiao, Q. Yang, C.-L. Wang, Y.-L. Fan, X.-F. Wu, and K.-J. Zhao, "Modification of plant height via RNAi suppression of OsGA20ox2 gene in rice," Euphytica, vol. 158, no. 1-2, pp. 3545, 2007.

[39] C. Zhu, L. Bortesi, C. Baysal et al., "Characteristics of Genome Editing Mutations in Cereal Crops," Trends in Plant Science, vol. 22, no. 1, pp. 38-52, 2017. 


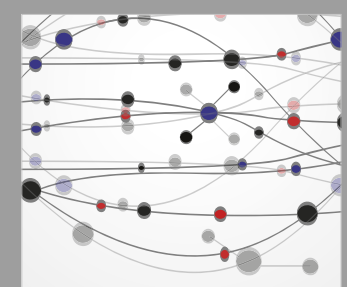

The Scientific World Journal
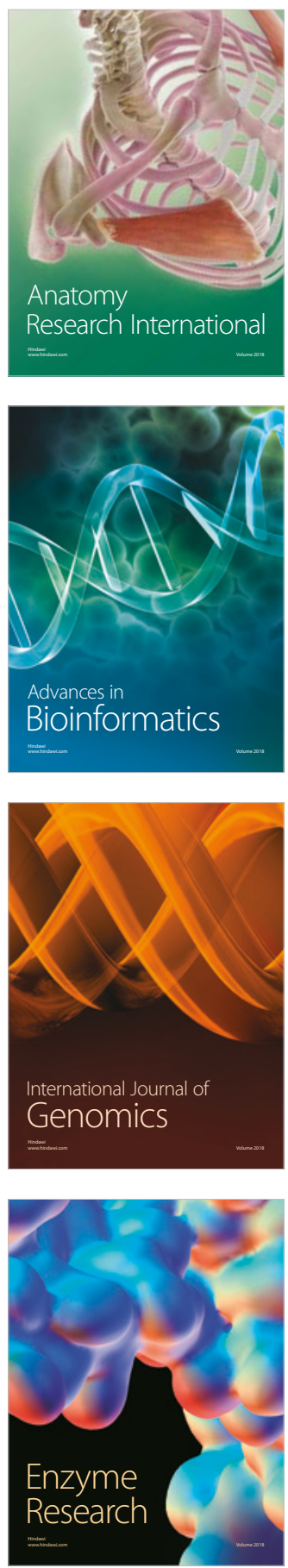
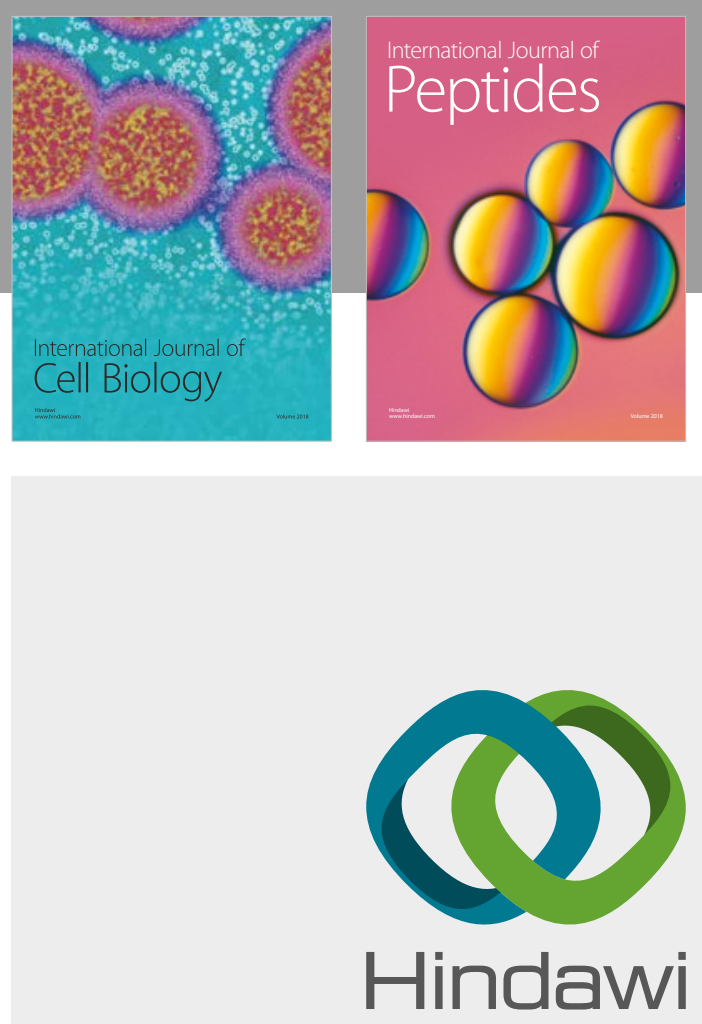

Submit your manuscripts at

www.hindawi.com
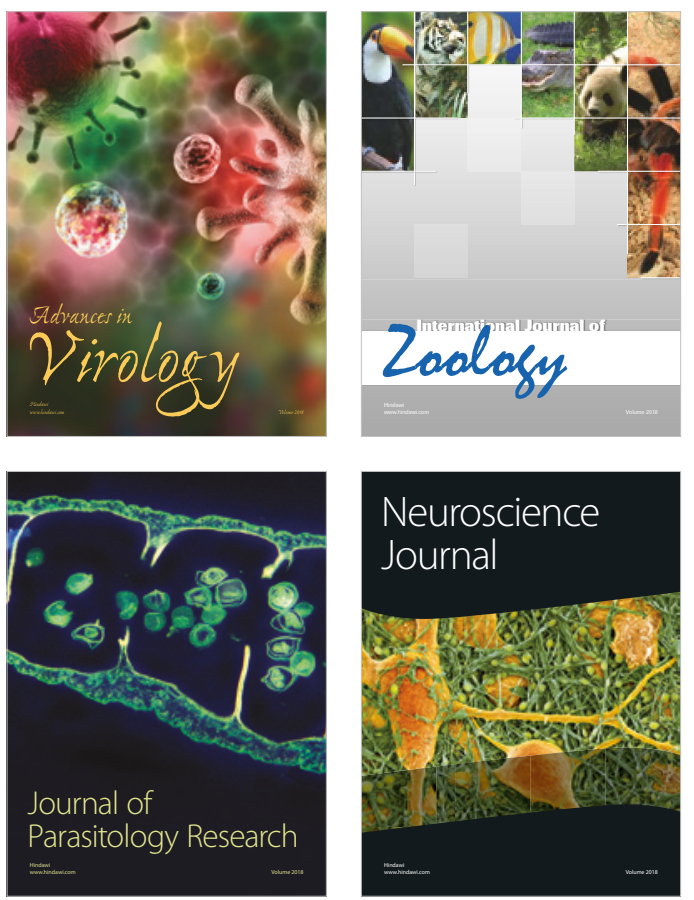
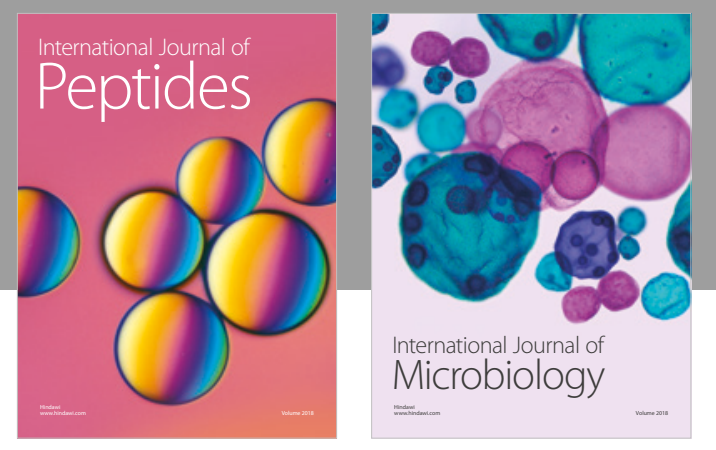

nternational Journal of Microbiology
Journal of
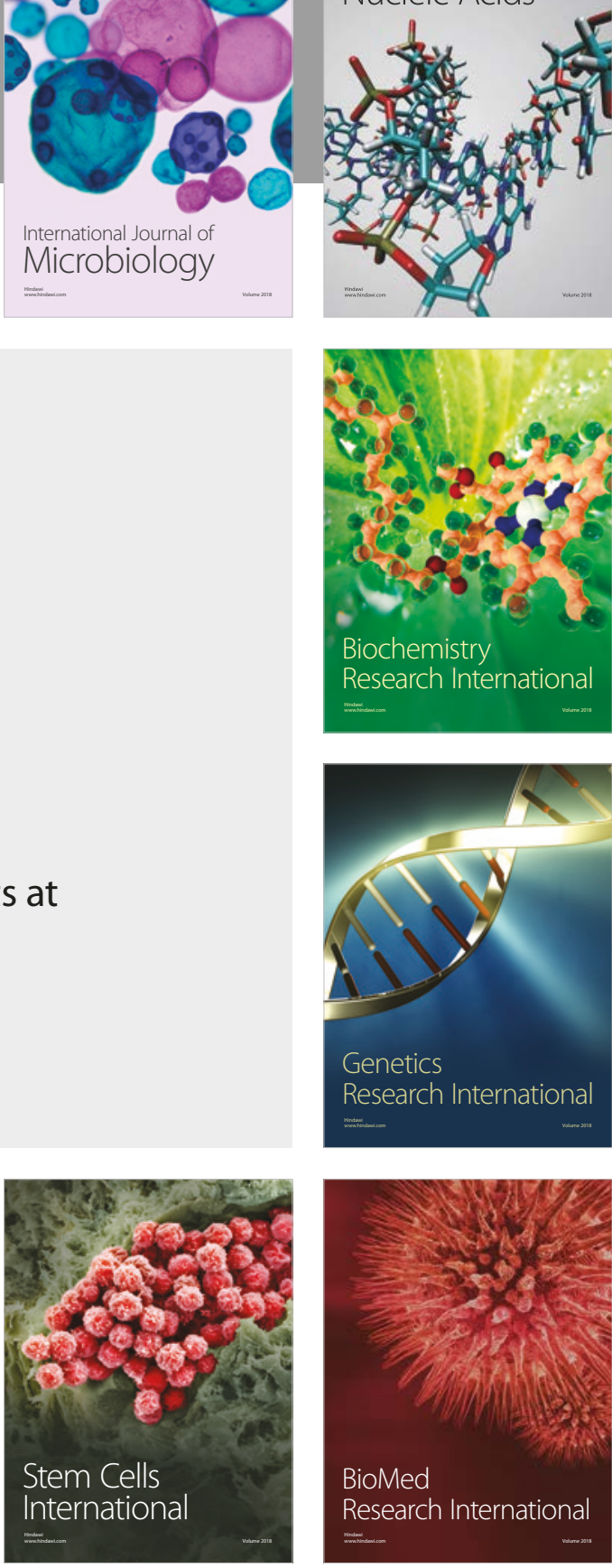
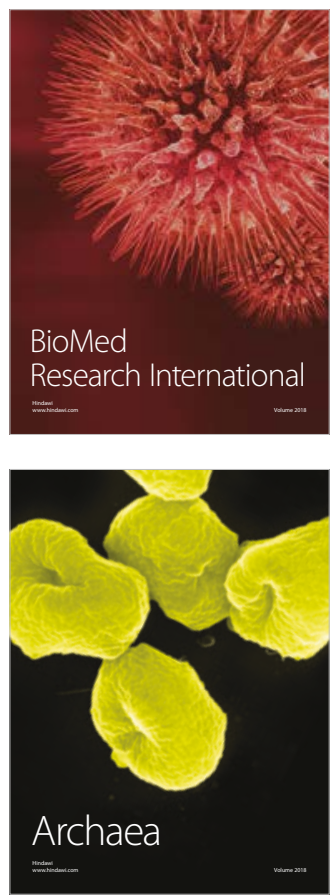